\title{
Removing the Ceiling on Droplet Microfluidics
}

Jian Wei Khor, ${ }^{1, *}$ Ulri N. Lee, ${ }^{1, *}$ Jean Berthier, ${ }^{1}$ Erwin Berthier, ${ }^{1, \#}$ and Ashleigh B. Theberge ${ }^{1,2, \#}$

${ }^{1}$ Department of Chemistry, University of Washington, Box 351700, Seattle, Washington 98195, United States

${ }^{2}$ Department of Urology, University of Washington School of Medicine, Seattle, Washington 98105, United States

* Equal contribution

${ }^{\#}$ Co-corresponding authors

\begin{abstract}
We developed an open channel droplet microfluidics system that autonomously generates droplets by utilizing competing hydrostatic and capillary pressure. With only our open channel PTFE device, pipettes, and commercially available carrier fluid, we produce up to hundreds of microliter droplets; tubing, electronics, or pumps are not required, making droplet technology feasible for research labs without external flow generators. Furthermore, we demonstrated conceptual applications that showcase the process of droplet generation, splitting, transport, incubation, mixing, and sorting in our system. Unlike conventional droplet microfluidics, the open nature of the device enables the use of physical tools such as tweezers and styli to directly access the system; with this, we developed a new method of droplet sorting and transfer that capitalizes on the Cheerios effect, the aggregation of buoyant objects along a liquid interface. Our platform offers enhanced usability, direct access to the droplet contents, easy manufacturability, compact footprint, and high customizability.
\end{abstract}

\section{Introduction}

We show for the first time droplet generation by an open microfluidic channel using passive forces alone; we also demonstrate downstream manipulations that are uniquely enabled by the open nature of the system. This work has tremendous potential to open new avenues in droplet microfluidics, a field which has grown immensely in the past decade. ${ }^{1-11}$ Droplet microfluidics is a powerful technology that uses microliter to picoliter droplets as chambers to conduct biological or chemical analyses and has many important applications in DNA sequencing, directed evolution, materials chemistry, and chemical reactions. ${ }^{1-11}$ Droplet microfluidics is an attractive technology because it is high throughput, miniaturizes chemical and biological processes, reduces reagent waste, and enables the use of precious or expensive reagents. Additionally, droplet manipulations such as sorting, mixing, and splitting are empowering for expanding potential applications of droplet microfluidics. ${ }^{1,2,5-11}$

In our system, we autonomously generate the droplets by leveraging the inherent hydrostatic pressure difference between the two immiscible fluids (the fluorinated carrier phase and aqueous phase), capillary pressure, and spontaneous capillary flow (SCF). Hydrostatic pressure is governed by the carrier fluid height (determined by the dimensions of the device) and density while capillary pressure is governed by interfacial tension, contact angle, and meniscus radius of curvature. SCF is a capillary flow that can be induced by balancing the wettability of the material and the dimensions of the channel through which the fluid is flowing. Additionally, SCF can be observed in open microfluidic or mesofluidic channels where at least one side of the channel is open to the air. ${ }^{12-17}$ Unlike conventional droplet microfluidics, our system has one side of the channel exposed, literally removing the ceiling to create an open air-liquid interface. The open surface of the channel gives access to the channel that conventional droplet microfluidics with closed, walled off channels does not provide; for example, a researcher can directly pipette into the channel or add/retrieve 
droplets, solid objects such as magnetic beads, or tissue samples. Our method enables the researcher to deliver the aqueous phase, followed by the carrier phase, each in a single pipetting step, to initiate the generation of droplets; there is no need for continuous pipetting or monitoring as in prior systems. ${ }^{18,19}$ Our SCF-driven droplet generation method eliminates the need for external flow generators, expanding the accessibility of droplet microfluidics to labs without flow generators and opening up possibilities for novel applications that are not feasible in devices requiring electricity.

Previous work in open droplet microfluidics includes the analysis of different modes of immiscible fluid plug flow in an open channel with various solvents as carrier fluid. ${ }^{20}$ Explorations of droplet manipulations (splitting, incubating, and merging) possible in an open channel were investigated where the droplets were pipetted by hand into the channel at specified locations prior to addition of the carrier fluid. ${ }^{21}$ Additionally, liquid handling systems have been used with open systems to keep flow rates constant for investigations of shear on cells in a hanging droplet ${ }^{22}$ and to generate droplets in open channels with syringe pumps. ${ }^{23}$ Our open channel droplet generator presented here is distinct from this prior work in that it shows for the first time the spontaneous generation of droplets in an open channel without any externally applied flow and without the direct actuation of a pipette to generate each droplet. Furthermore, in contrast to prior systems, ${ }^{20,21}$ the carrier fluid used to generate and transport droplets in our system (HFE fluorinated oil) is commonly used for biological applications of droplet microfluidics, thus making our open droplet system compatible for biological experiments..$^{2,5,7,8,24,25}$ The work of Soitu et al. ${ }^{26-29}$ and Li et al. ${ }^{30-33}$ highlighted the importance of manipulations of droplets for cell biology in their open systems where droplets were formed under an immiscible phase by segmenting aqueous solution with a physical stylus and simple pipetting. Our work is complementary to these two methods because our droplets are selfgenerating in an open system where they can then be manipulated for biological applications. Other methods to generate droplets using pipettes include simple agitation of a biphasic solution either with or without cavity-containing microparticles. ${ }^{18,34}$

Herein we describe a system that achieves autonomous droplet generation of up to hundreds of droplets in an open microfluidic channel. We made this possible with only the open channel, pipettes, and commercially available carrier fluid; tubing, electronics, or pumps are not required, making droplet technology feasible for research and biology labs without external flow generators. Additionally, we developed a theoretical model to describe the phenomenon observed. The model is derived from the pressure difference between hydrostatic pressures and capillary pressures across the aqueous plug, and a critical threshold for droplet generation is found. Both the experimental results and theoretical model show that interfacial tension, contact angle, and constriction width play critical roles in droplet generation. We also developed a suite of droplet manipulation techniques that capitalize on the open nature of our devices, including novel methods for moving droplets with a specialized stylus or tweezers to sort and transfer droplets within a device or into another device or well plate.

\section{Results and Discussion}

Autonomous droplet generation in an open microfluidic channel. Our open droplet generation platform demonstrates the ability to autonomously generate droplets without any midway intervention, and the open surface nature of our channel provides direct access to manipulate droplets (Figure 1). The operation of the device is simple; aqueous phase is first pipetted in the 
a

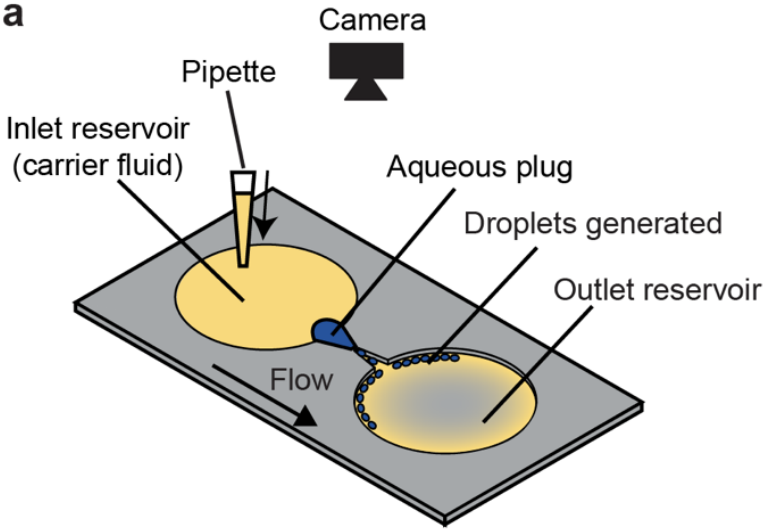

b

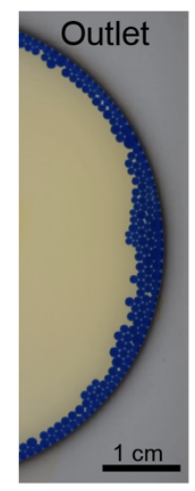

d
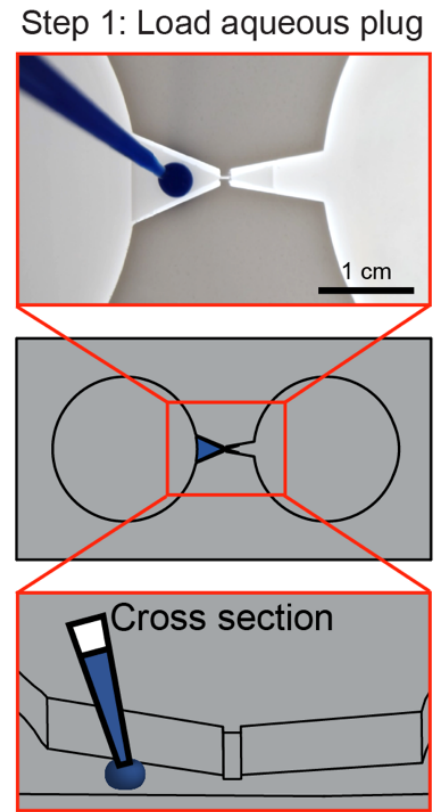

Step 2: Load carrier fluid
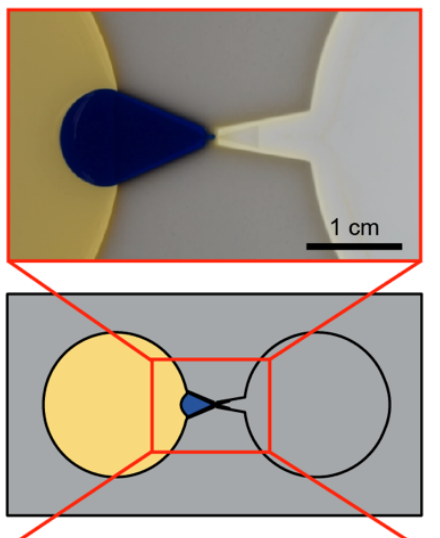

Cross section

C

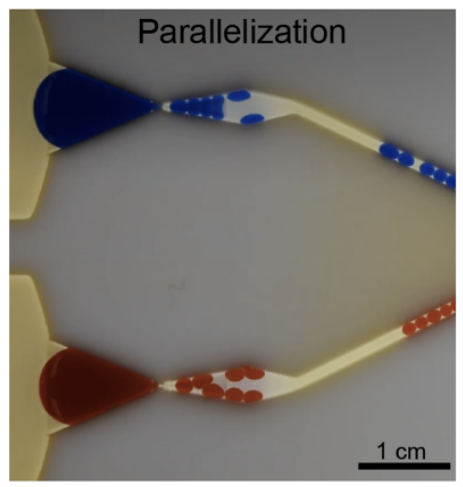

Step 3: Droplet generation

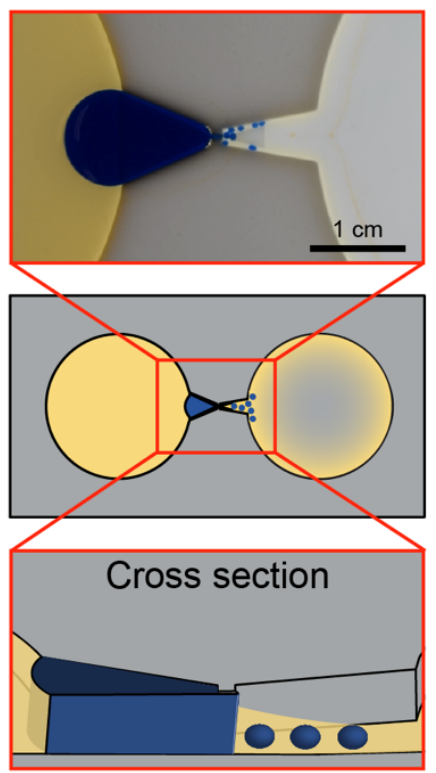

e

\section{Open microfluidics enables direct access to droplets}

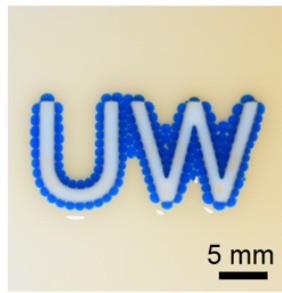

Patterning

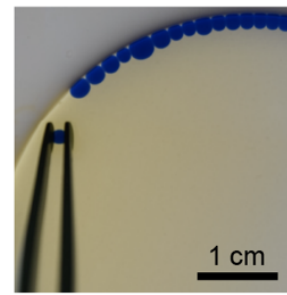

Transferring

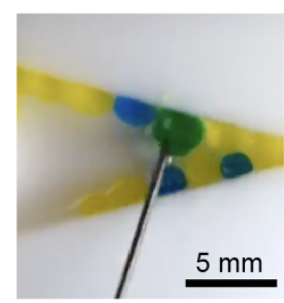

Merging

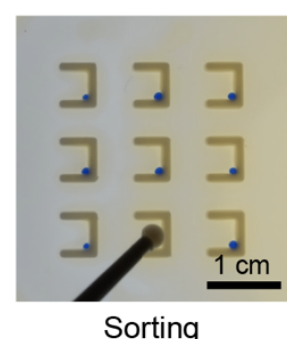

Sorting

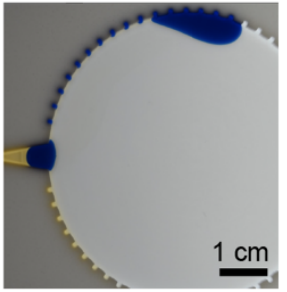

Splitting

Figure 1. Device design, workflow, and manipulation of droplets. a Schematic representation of the device, b image of generated droplets in the outlet reservoir, and $\mathbf{c}$ droplets generated in parallel. d Workflow for droplet generation using passive forces derived from pressure (hydrostatic pressure and capillary pressure) in an open device (Supplementary Video 1). e Droplet manipulations downstream (Supplementary Video 2-6). 
converging region where it is in full contact with the channel walls (Figure 1d, left). Next, the carrier fluid, a fluorinated oil, is pipetted in the inlet reservoir to establish a hydrostatic pressure that pushes the aqueous plug into the narrow constriction $(0.2 \mathrm{~mm}-3 \mathrm{~mm}$ in width) (Figure $1 \mathrm{~d}$, middle). As the anterior portion of the aqueous plug exits the narrow constriction, it pinches off into small droplets (Figure 1d, right).

There are five regions in the device; the origin of flow is at the (1) inlet reservoir which leads to the (2) converging region, followed by the (3) narrow constriction, (4) diverging region, and (5) outlet reservoir (Figure S1). The converging region holds the aqueous plug where the anterior end of the plug meets the narrow constriction. At the constriction, droplets are generated into the diverging region and flow to the outlet reservoir. The angles of the converging and diverging region ( $45^{\circ}$ and $20^{\circ}$ respectively) were found to generate the most consistent and most monodisperse droplets. Furthermore, a pair of square protrusions forms the narrow constriction which creates a large capillary pressure to oppose the hydrostatic pressure of the carrier fluid at the inlet reservoir. The hydrostatic pressure builds up as carrier fluid fills the inlet reservoir. At the narrow constriction and converging-diverging region there is a $0.2 \mathrm{~mm}$ tall step with grooves on both sides to allow carrier fluid to pass through the narrow constriction and prewet the channel walls prior to droplet generation which was found to be essential for continuous droplet generation. Prewetting of the channel walls by SCF occurs due to the low contact angle of the carrier fluid.

One critical breakthrough of this work is the use of PTFE as the channel material. Previously, in open channel droplet research, channels were fabricated from PMMA and could only transport droplets with organic solvents which is not ideal for applications of droplet microfluidics because organic solvents are often cytotoxic and known to extract small molecules from the droplets. ${ }^{20,21}$ The wetting properties of fluorinated oil, HFE 7500, on PTFE and the high contact angle of the aqueous plug allowed for droplet generation and manipulation (Figure 1) to occur in our channel. Fluorinated oils are ideal for life science applications in droplet-based microfluidics because of their biocompatibility - they have been used in applications ranging from human cell culture to digital droplet polymerase chain reaction (ddPCR). ${ }^{1-11,24}$ Finding a solution that does not require organic solvents was essential to furthering biological applications of our technology.

Condition for droplet generation. We find that droplet generation occurs when the aqueous plug overcomes the hydrostatic pressure difference and capillary pressure difference that allows it to enter the narrow constriction. The aqueous plug is pushed into the narrow constriction by the action of the hydrostatic pressure difference across the plug's interface. (Note: a non-wetting plug should move spontaneously away from convergent geometries when no external forces of parallel direction are applied, and the hydrostatic pressure resulting from the carrier fluid is able to counteract this tendency). ${ }^{13}$ Based on empirical observation from high-speed videos, the droplet formation process (plug advancement, bulbing, thread formation, and pinch off) is similar to droplet formation in closed system droplet microfluidics (Figure $2 \mathrm{a}$ and $b$ ). ${ }^{3-5,35}$ The anterior portion of the aqueous plug is initially settled at the narrow constriction. As the anterior portion of the aqueous plug is advancing into the narrow constriction (plug advancement phase) it forms a bulbous shape (bulbing phase). Next, a liquid thread is formed (thread formation phase in Figure $2 \mathrm{a}$ and red arrow depicting the thread in Figure $2 \mathrm{~b}$ ), and the liquid thread diameter decreases until it pinches off and droplet formation occurs (pinch off phase).

Figure $2 \mathrm{~b}$ shows the droplet formation process for various surfactant concentrations, $\mathrm{c}$, and constriction widths, w. We observed that the phases of the droplet formation process are nearly 


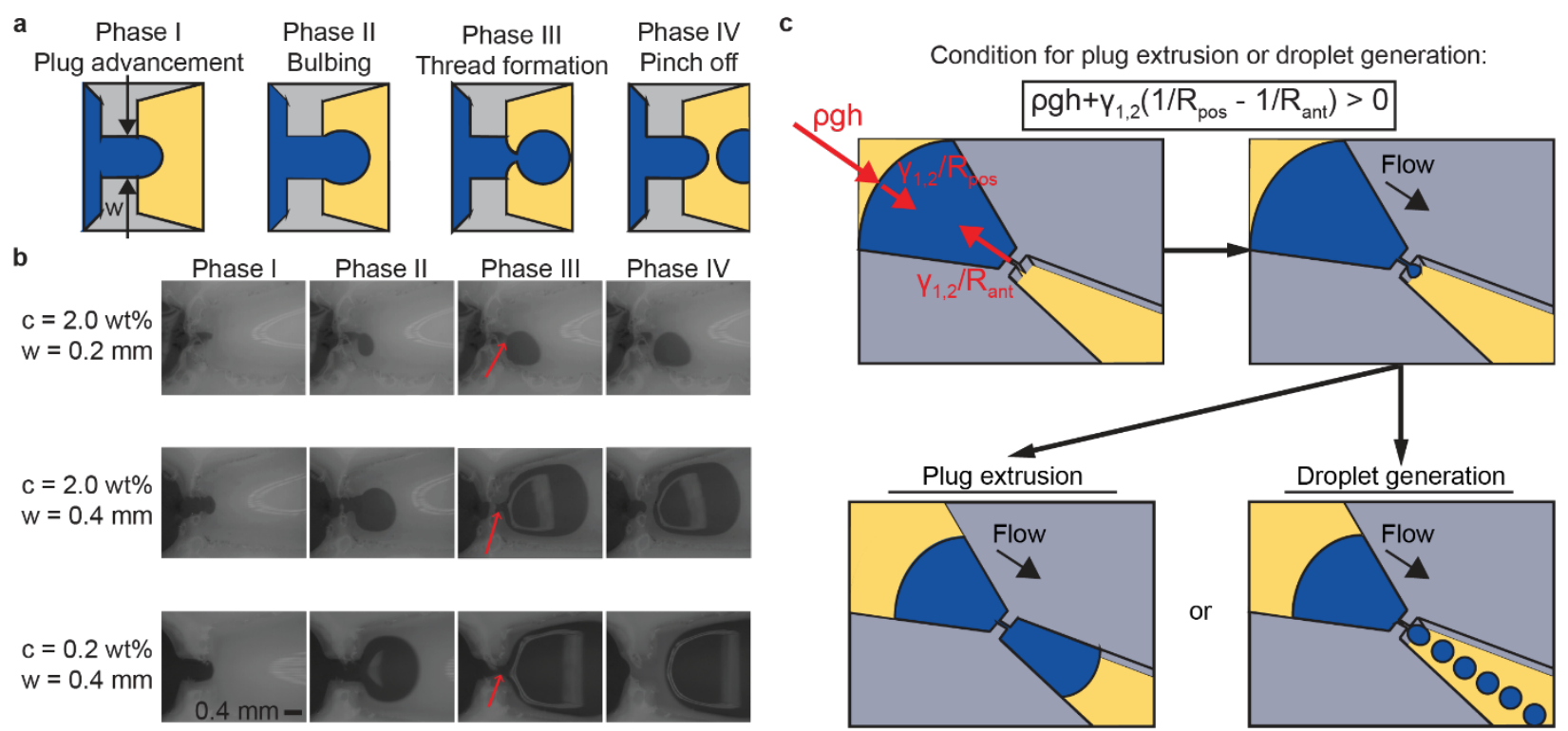

Figure 2. Snapshots of droplet formation with a high-speed camera and geometry of the open channel constriction for droplet generation. a Schematic of the phases observed during droplet formation. b Droplet formation in the constriction at various constriction width and surfactant concentration. Red arrow depicts the thread formation. c Hydrostatic pressure overcoming capillary pressure resulting in the aqueous plug being extruded or generating droplets at the constriction.

identical across the various surfactant concentrations and constriction widths used in this study (Figure 2b). We also observe that the constriction geometry and surfactant concentration is a determining factor in the final droplet volume generated. For the aqueous plug interface to extend past the narrow constriction, the hydrostatic pressure, $\rho g h$ at the posterior side of the plug has to overcome the capillary pressure, $\gamma_{1,2}\left(1 / R_{\text {pos }}-1 / R_{\text {ant }}\right)$ (Figure $\left.2 \mathrm{c}\right)$. Then, two possible outcomes occur: plug extrusion or droplet generation. We define plug extrusion as the whole aqueous plug extruding out of the constriction and droplet generation as a consistent generation of droplets that have relative standard deviation of generated droplet volume between $5 \%-7 \%$. Note that plug extrusion sometimes leads to the plug breaking into two or three segments when exiting the diverging region. The pressure difference when the aqueous plug interface extends past the narrow constriction is:

$$
\rho g h+\gamma_{1,2}\left(\frac{1}{R_{\text {pos }}}-\frac{1}{R_{\text {ant }}}\right)>0(1)
$$

where $g$ is the gravitational acceleration, $\gamma_{1,2}$ is the interfacial tension between carrier fluid and aqueous plug, $\rho$ is the carrier fluid density, $R_{\text {pos }}$ is the radius of curvature of the posterior plug interface, and $R_{\text {ant }}$ is the radius of curvature of the anterior plug interface. The LHS of Eq. (1) has to be greater than 0 for the aqueous plug interface to extend past the narrow constriction.

By rearranging the pressure difference involving the hydrostatic pressures and capillary pressures, the condition for the aqueous plug extrusion or droplet generation is (for the theoretical derivation, please see SI Supplementary Note 1): 


$$
w>\frac{2 \gamma_{1,2}\left|\cos \theta_{1,2, s}\right|}{\rho g h}
$$

where $\mathrm{w}$ is the constriction width, $\theta_{1,2, s}$ is the contact angle between aqueous plug, carrier fluid, and channel wall, and $h$ is the carrier fluid height. Based on experimental observation, the aqueous plug height is equal to the carrier fluid height.

We display the theoretical threshold for when the aqueous plug extrusion or droplet generation occurs in a regime map (Figure 3a dotted line, from Eq. (2)). In Figure 3a, we observed empirically that there exists a well-defined region (teal shaded region) where droplet generation occurs when constriction width is small ( $\mathrm{w} \leq 0.6 \mathrm{~mm}$ ) and the condition in Eq. (2) is met (discussion on units for Eq. (2) can be found in SI Supplementary Note 2). In the droplet generation region (teal shaded region), the constriction width, interfacial tension $\gamma_{1,2}$, and contact angle $\theta_{1,2, s}$ are the three factors that determine the volume of the droplets formed when the channel height and carrier fluid density remain constant. The $\gamma_{1,2}$ and $\theta_{1,2, s}$ is varied by the concentration of the surfactant, c. We observed that at a constriction width of $0.2 \mathrm{~mm}$, when $\gamma_{1,2}$ and $\theta_{1,2, s}$ increases (due to lowering fluorinated surfactant (FS) concentration from $2.0 \mathrm{wt} \%$ to $0.2 \mathrm{wt} \%$ or lower), the anterior portion of the aqueous plug cannot enter the narrow constriction and no plug extrusion occurs (Figure $3 b$ ). However, the aqueous plug can extrude from the narrow constriction if the constriction width increases to $0.4 \mathrm{~mm}$ at a FS concentration, c, of $0.2 \mathrm{wt} \%$. This observation matches Eq. (2) and the regime map at Figure 3a; when $\gamma_{1,2}$ and $\theta_{1,2, s}$ increases, the minimum width that allows plug extrusion is larger as well. Note that plug extrusion is possible without droplet generation ( $\mathrm{w}=3.0$ $\mathrm{mm}, \mathrm{c} \geq 0.2 \mathrm{wt} \%)$. At the highest $\gamma_{1,2}$ and $\theta_{1,2, s}(0.0 \mathrm{wt} \% \mathrm{FS})$, the anterior portion of the aqueous plug cannot enter the narrow constriction for any of the

a

Condition for plug extrusion or droplet generation: $\mathrm{w}>2 \mathrm{\gamma}|\cos \theta| / \rho g h$

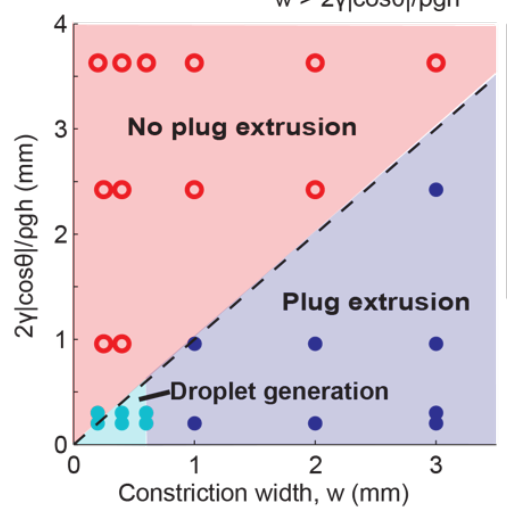

b

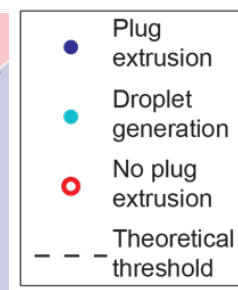

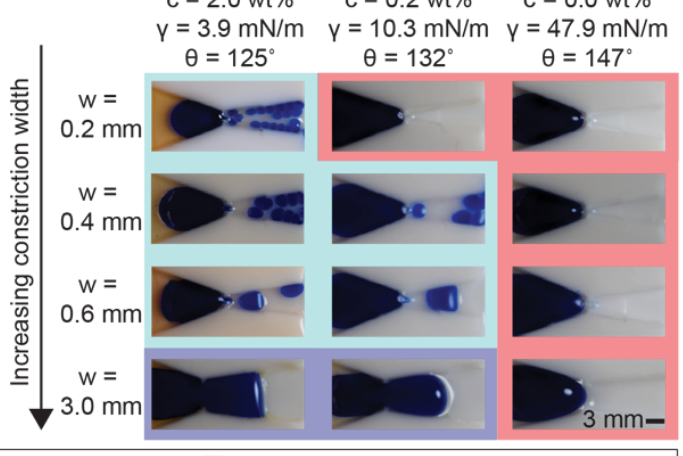

Plug extrusion Droplet generation No plug extrusion

Figure 3. Conditions for plug extrusion and droplet generation. a Regime map of plug extrusion and droplet generation with theoretical threshold (dashed line per Eq. (2)); experimental data points of plug extrusion (purple filled circle), droplet generation (teal filled circle), and no plug extrusion (red open circle). The regions are plug extrusion (purple shaded region), droplet generation (teal shaded region), and no plug extrusion (red shaded region). b Montage of plug extrusion and droplet generation when varying constriction width, fluorinated surfactant concentration (c, wt \%), interfacial tension $\gamma_{1,2}$, and contact angle $\theta_{1,2, s}$. 
constriction width dimensions tested, thus plug extrusion does not occur (Figure 3b, consistent with the phase diagram in Figure 3a).

Figure 4 depicts the effect of surfactant concentration ( $c=2 \mathrm{wt} \%$ and $\mathrm{c}=0.2 \mathrm{wt} \%)$ and constriction width $(\mathrm{w}=0.2 \mathrm{~mm}$ and $\mathrm{w}=0.4 \mathrm{~mm}$ ) on generated droplet volume (data point presented as mean $\pm \mathrm{SD}, \mathrm{N} \geq 90$ ). The droplet volume generated increases with increasing constriction width $\mathrm{w}$, interfacial tension $\gamma_{1,2}$, and contact angle $\theta_{1,2, s}$ (decreasing surfactant concentration). The smallest droplet volume generated for $\mathrm{w}=0.2 \mathrm{~mm}$ is $0.52 \pm 0.025 \mu \mathrm{L}$ and $\mathrm{w}=0.4 \mathrm{~mm}$ is $2.49 \pm 0.175 \mu \mathrm{L}$. Interestingly, the relative standard deviation of our droplet generation method ranges between 5\% and $7 \%$ while conventional methods are $1 \%-3 \%{ }^{35,36}$ This shows that the dispersity of our system is relatively close to conventional methods. We believe the relative standard deviation can be reduced by having better control of the hydrostatic pressure. Future redesigns will aim to maintain the hydrostatic pressure to be relatively constant. We also note that the physics underlying the droplet formation process is scalable to smaller length scales, therefore smaller droplet volumes will be achievable in future work if alternative fabrication processes such as advanced CNC milling or cleanroom fabrication processes are used to create devices with smaller constriction widths. Here, we chose $0.2 \mathrm{~mm}$ as the minimum constriction width based on the limitations of our current $\mathrm{CNC}$ milling process.

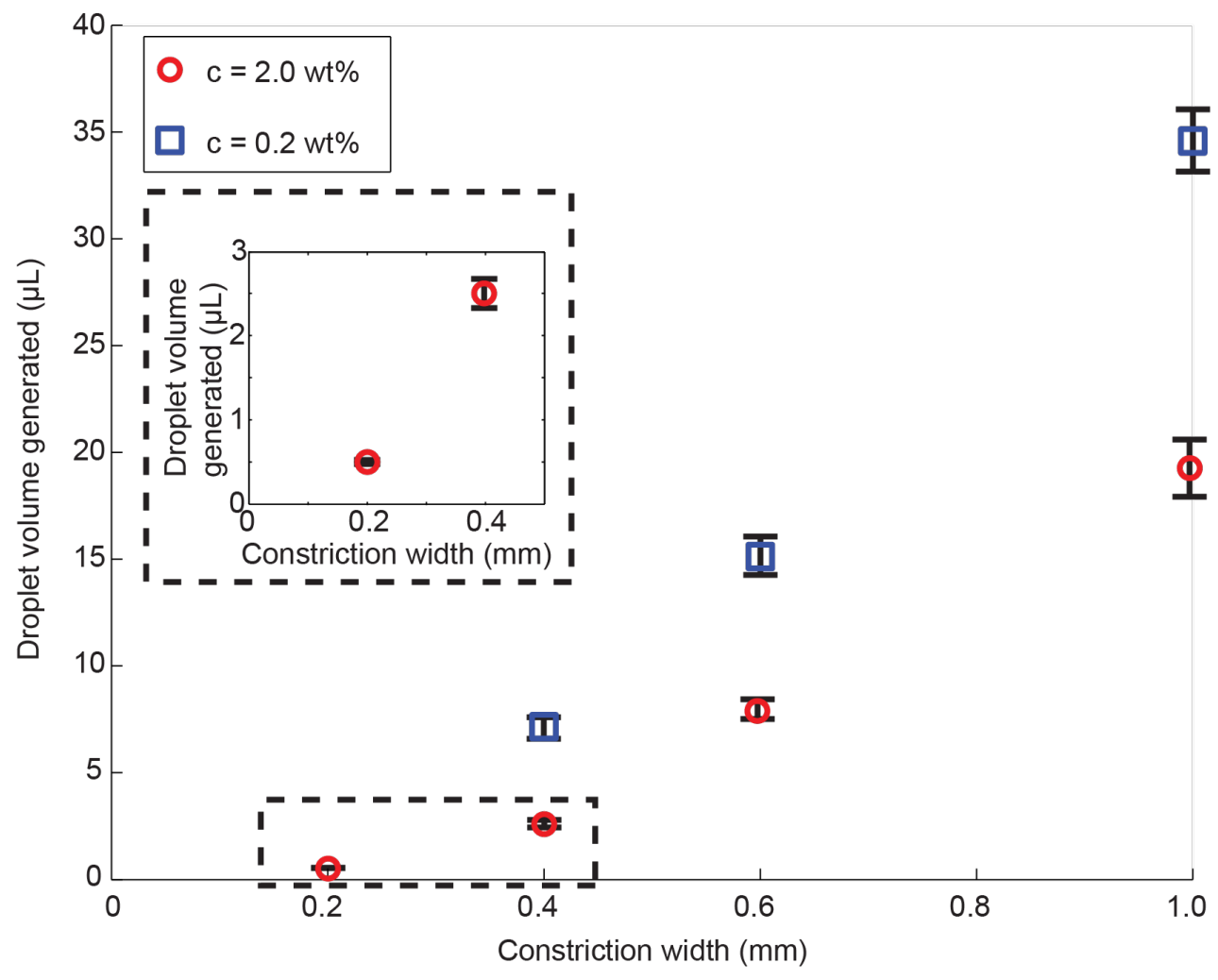

Figure 4. Droplet volume varies with constriction width and surfactant concentration. Plot of droplet volume generated when varying surfactant concentration and constriction width (data point is plotted as mean $\pm \mathrm{SD}, \mathrm{N} \geq 90$ droplets). 
Downstream droplet manipulation in open microfluidic devices. Droplet manipulation is an important component of utilizing droplet microfluidics as a tool to study biology and chemistry. In this work, droplet manipulation methods are demonstrated in an open microfluidic device to present the possibilities of conducting future biological and chemical studies with this device. The open surface of the device enables us to create new methods to physically move the droplets. For example, we developed methods enabling us to reach into the device with specialized tweezers or a needle to move or mix targeted droplets on demand, with droplets chosen in real-time at the discretion of the researcher. Here, we demonstrate droplet manipulations and workflows for chemical reactions followed by droplet sorting that are enabled by open microfluidics.

a

Cheerios effect overview

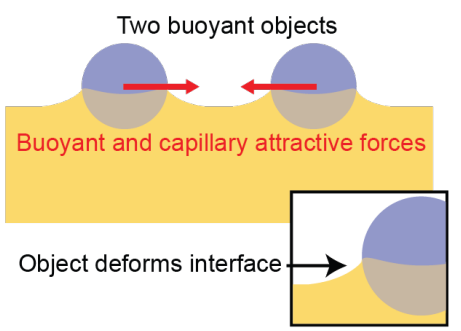

Cheerios effect with PTFE tweezers

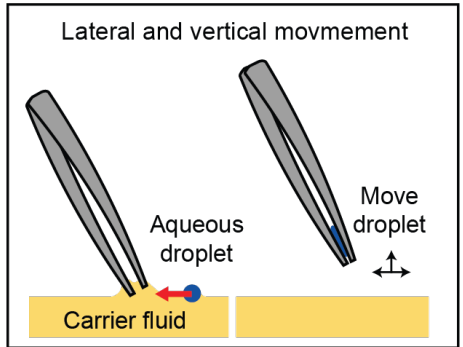

Cheerios effect with PTFE stylus

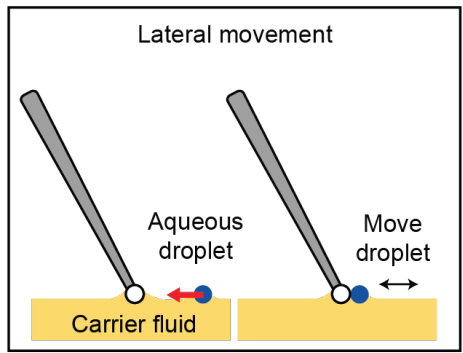

b Picking up and transferring droplets with PTFE tweezers

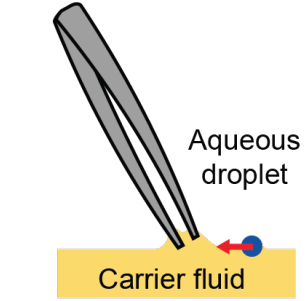

Droplet moves toward tweezers

C

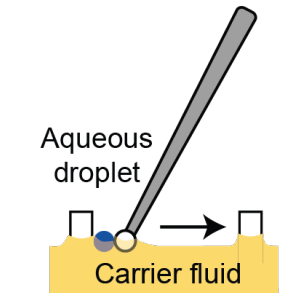

Droplet is removed from wall

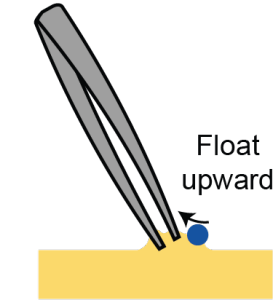

Droplet floats up the interface

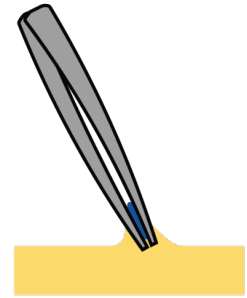

Droplet is captured by tweezers

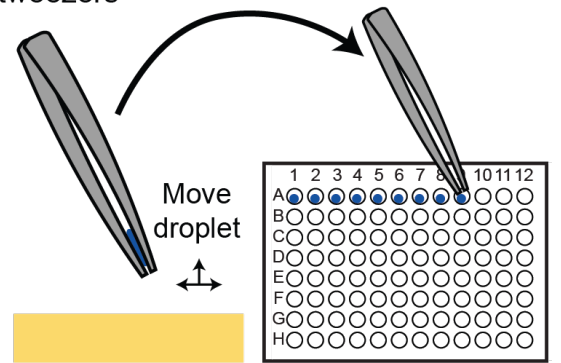

Droplet is lifted out of carrier fluid and transferred to well plate

Figure 5. The Cheerios effect enables droplet transport. a Two neighboring buoyant objects aggregate due to the Cheerios effect. ${ }^{37,38}$ The buoyant object deforms the surface of the liquid resulting in a rising interface, and when a second buoyant object is near it tends to float up the rising interface. The tweezers and PTFE stylus behave similarly because they also deform the surface so the droplets tend to aggregate towards them. b Picking up droplets from an open microfluidic device and transferring them to another location (e.g., well plate) with PTFE-coated tweezers. c Transporting droplets from one wall to another with a PTFE ball mounted on a stylus. 
Droplet transport and droplet sorting is an integral component of droplet microfluidics workflows. We developed a new method to selectively transfer single droplets to locations both on and off the open droplet generating device by picking them up with PTFE-coated tweezers or moving them with a PTFE stylus. The PTFE-coated tweezers and styli transport droplets laterally by utilizing the Cheerios effect, which is the phenomenon of multiple objects aggregating due to the objects deforming the liquid interface (Figure 5a). ${ }^{37-41}$ The PTFE-coated tweezers and styli are placed at the surface of the carrier fluid, causing the carrier fluid to wet the tweezers or stylus and form a rising interface. The droplets are buoyant and when they are near the meniscus created by the tweezers or stylus they then move toward the upward rising fluid interface. Additionally, once the droplet enters between the tweezers, the droplet rises up the tweezers due to the tendency for droplets to move towards divergent geometries (Figure 5b). ${ }^{13}$ This allows droplets to be picked up and transferred to another location (e.g., well plate). To detach the droplet from the tweezers or stylus, the tweezers or stylus is abruptly moved away from the droplet once it reaches the final location and is detached due to inertia. Further, the meniscus formed by the presence of a wall or other micromilled feature (Figures $5 \mathrm{c}, 6 \mathrm{~b}$, and $6 \mathrm{c}$ ) causes the droplet to again move up the rising interface to rest on the wall.
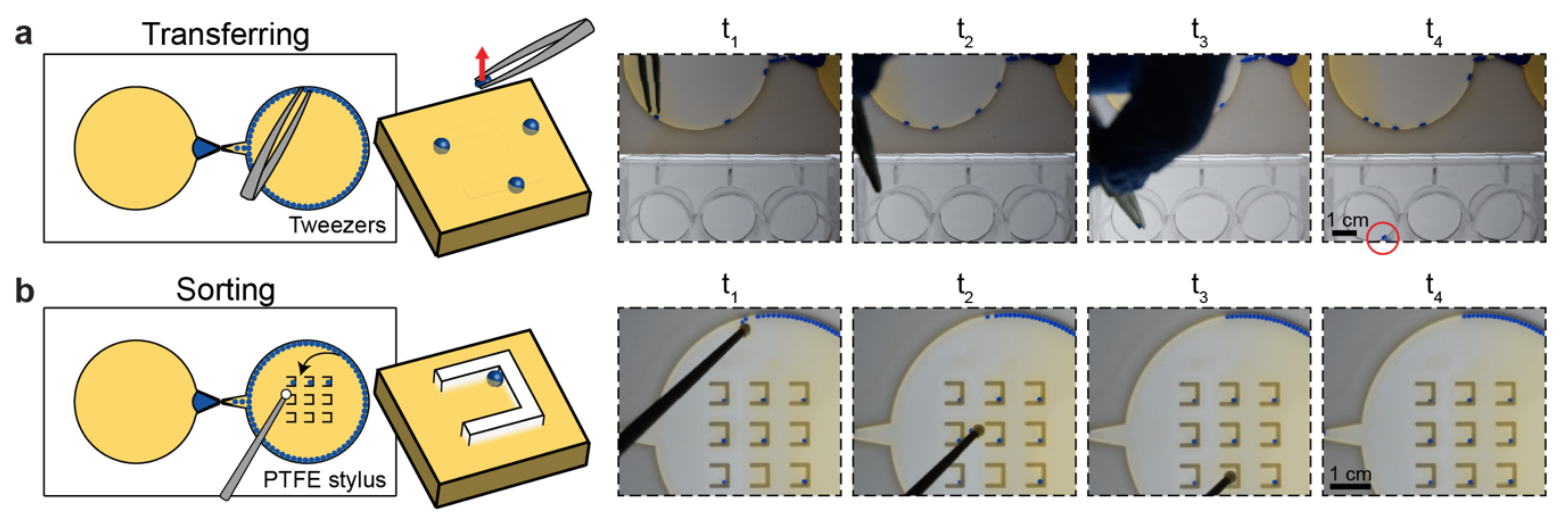

$\mathrm{t}_{2}$

$t_{3}$
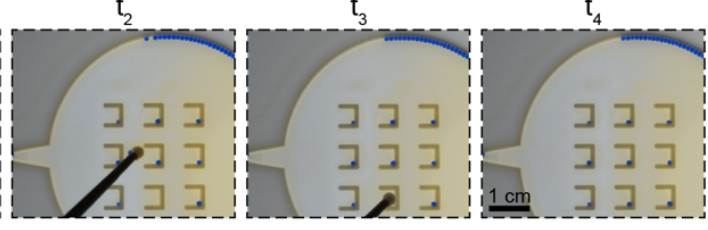

C
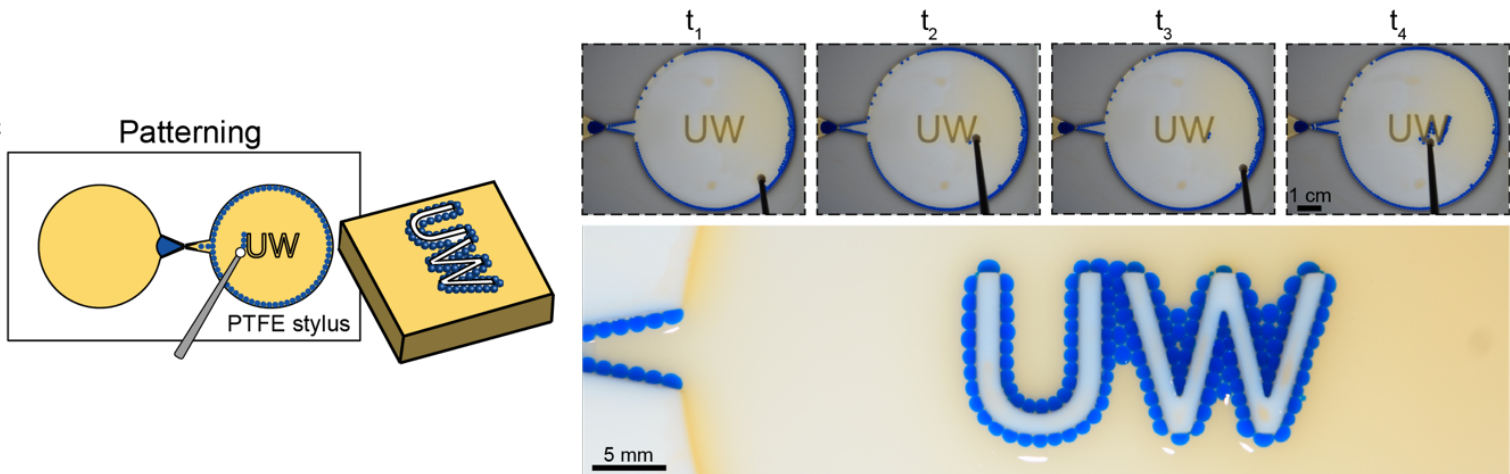

Figure 6. Open surface of the open microfluidic channel enables on-demand direct manipulation of droplets. a Selectively retrieving droplets from an open microfluidic device and transferring them to a well plate with PTFE-coated tweezers (Supplementary Video 3). b Sorting droplets into individual chambers with a PTFE ball mounted on a stylus (Supplementary Video 5). c PTFE stylus is used to transfer droplets to the "UW" pillar (Supplementary Video 2). 
PTFE is important for droplet transport because the PTFE prevents the aqueous droplet from strongly adhering to the tweezers, making release of the droplet easy. Using a similar mechanism, we use a stylus with a PTFE bead adhered to the tip to gently move droplets around the reservoir for sorting and patterning droplets (Figure $6 \mathrm{~b}$ and $6 \mathrm{c}$ ) enabling the droplets to be sorted (on demand). To summarize, the PTFE stylus enables lateral droplet transport and the PTFE-coated tweezers enables both lateral droplet transport and vertical droplet transport (i.e., picking up droplets). In future work, this approach could be used to sort droplets based on a reaction outcome or transfer droplets to a different part of a chip for a multistep process.

While we focused on moving individual droplets to highlight the ability to move droplets selectivity, this method would be simple to multiplex using an array of styli (for example, a threepronged stylus could be used to move three droplets at once from the columns of the array similar to Figure 6c). The direct access to the droplets is unique to our open channel systems and allows users to directly extract and/or manipulate the contents of the channel. This is enabling for users who cannot rely on power-heavy solutions in conventional droplet microfluidics like acoustophoresis, electrophoresis, and electrowetting-on-dielectric (EWOD) or users who would like the flexibility to manipulate droplets at any point in their device, without the need to prepattern electrodes. ${ }^{10}$ At this same time, for applications requiring automation, this method could be automated by mounting the stylus on a robotic XYZ controller interfaced with a fluorescent or colorimetric readout in the droplets to facilitate sorting based on a chemical/biochemical readout. The ability to move droplets flexibly and on demand - both on- and off-chip — shows great promise for being able to conduct multistep chemical and biological experiments and incorporating analytical readouts that are best implemented outside of the original microfluidic chip embodiment.

In Figure 6c, the generated droplets are patterned by transporting them toward the "UW" pattern using a PTFE bead to outline the pattern. It is important to note that droplets were able to be transported to sharp corners of the "W" with ease using the PTFE bead. In contrast, traditional droplet microfluidics would require pumps and valves to change the trajectory of the droplets. Furthermore, our channel design is not constrained by requiring prior knowledge of the location to which the droplets will be transported. This allows our channel design to be modular by being able to continuously upgrade or add new patterns (e.g., replacing "UW" pattern with another pattern) directly on the channel. Essentially, open microfluidics simplifies droplet microfluidics by making it user-friendly, customizable, and adaptable for integration of physical probes and tools downstream.

The ability to take small aliquots of a droplet after it is generated is useful for downstream analysis or processing steps, particularly when working with small volumes of precious reagents. ${ }^{1-11} \mathrm{We}$ demonstrated that we can split smaller droplets off from a large aqueous plug with a crenulation feature along the outlet reservoir wall (Figure 7a). In this case, the constriction allows aqueous plug to extrude and flow along the crenulations. As the plug travels along the crenulations via SCF, it fills the crenulation, and as the posterior end of the large plug fully passes the crenulation it shears off leaving the crenulation filled with a small droplet. The droplets in the crenulations can be retrieved with a pipette. Note that during plug extrusion, the plug sometimes breaks into two or three segments when exiting the diverging region. 

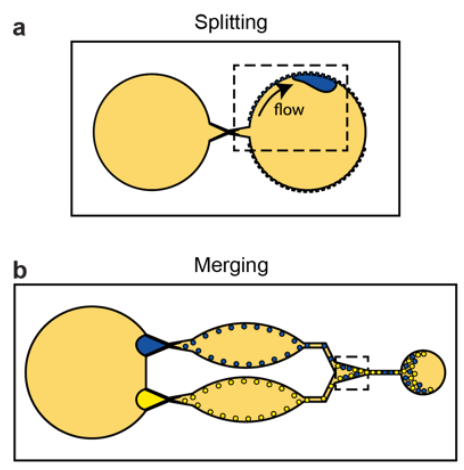
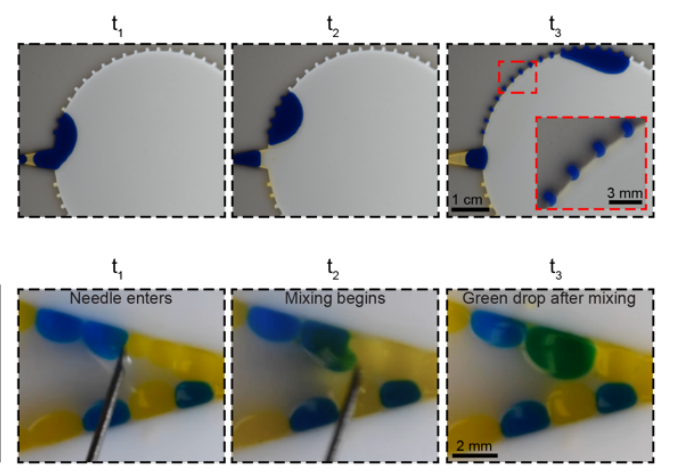

Figure 7. Splitting and merging droplets in an open droplet generator. a Crenulations along the outlet reservoir capture portions of an aqueous plug, and as the plug passes the crenulation, a small droplet splits off and remains in the crenulation (Supplementary Video 6). b A yellow and blue droplet are merged by mixing with a needle to form a green droplet (Supplementary Video 4).

In Figure $7 b$, two droplet merging is demonstrated by disrupting the interface of the droplets using a simple needle. The needle is swirled between the two droplets to disrupt the interface between the droplets resulting in their mixing into one. This merging technique provides versatility that is unprecedented in prior droplet fusion methods (such as electrofusion or fusion based on electrowetting on dielectric), which often require the placement of specific components (e.g., electrodes) in the device design. ${ }^{1,10}$ In contrast, our new fusion method will allow users to induce fusion on demand at any location in the open device; the location need not be decided prior to the experiment. In future work, the merging step could also be automated using a needle mounted on an XYZ controller as described for the droplet transport with tweezers and styli mentioned above.

Droplet merging opens up applications for chemical reactions, biochemical assays, and other multi-reagent processes. Selective droplet merging is demonstrated in Figure 8 using droplets of $\mathrm{KSCN}$ and $\mathrm{Fe}\left(\mathrm{NO}_{3}\right)_{3}$ mixed with blue dye for visualization. Droplets of the two reagents are formed using two parallel open-channel droplet generators. Then, two droplets are randomly selected from the outlet reservoir and transported to a platform with a pair of PTFE-coated tweezers (yielding three possible combinations: two droplets containing KSCN, two droplets containing $\mathrm{Fe}\left(\mathrm{NO}_{3}\right)_{3}$, or a droplet containing $\mathrm{KSCN}$ and a droplet containing $\left.\mathrm{Fe}\left(\mathrm{NO}_{3}\right)_{3}\right)$. On the platform, the droplets are merged by disrupting the interfaces of the droplet pair with a needle. When a droplet containing $\mathrm{KSCN}$ and a droplet containing $\mathrm{Fe}\left(\mathrm{NO}_{3}\right)_{3}$ merge, they change color from blue to red due to the formation of $\mathrm{Fe}(\mathrm{SCN})_{3}$. Merged droplets are then sorted to chambers categorized by reacted and unreacted droplets using PTFE-coated tweezers. The purpose of this proof-of-concept workflow is to demonstrate the ability to perform a reaction and sort droplets based on "hits" (in this case droplets containing the reaction product $\left.\mathrm{Fe}(\mathrm{SCN})_{3}\right)$. This suggests the potential for conducting droplet sorting tasks in applications like single cell encapsulation, directed evolution of enzymes, and process optimization of synthetic reactions to synthesize small molecules or novel materials. 


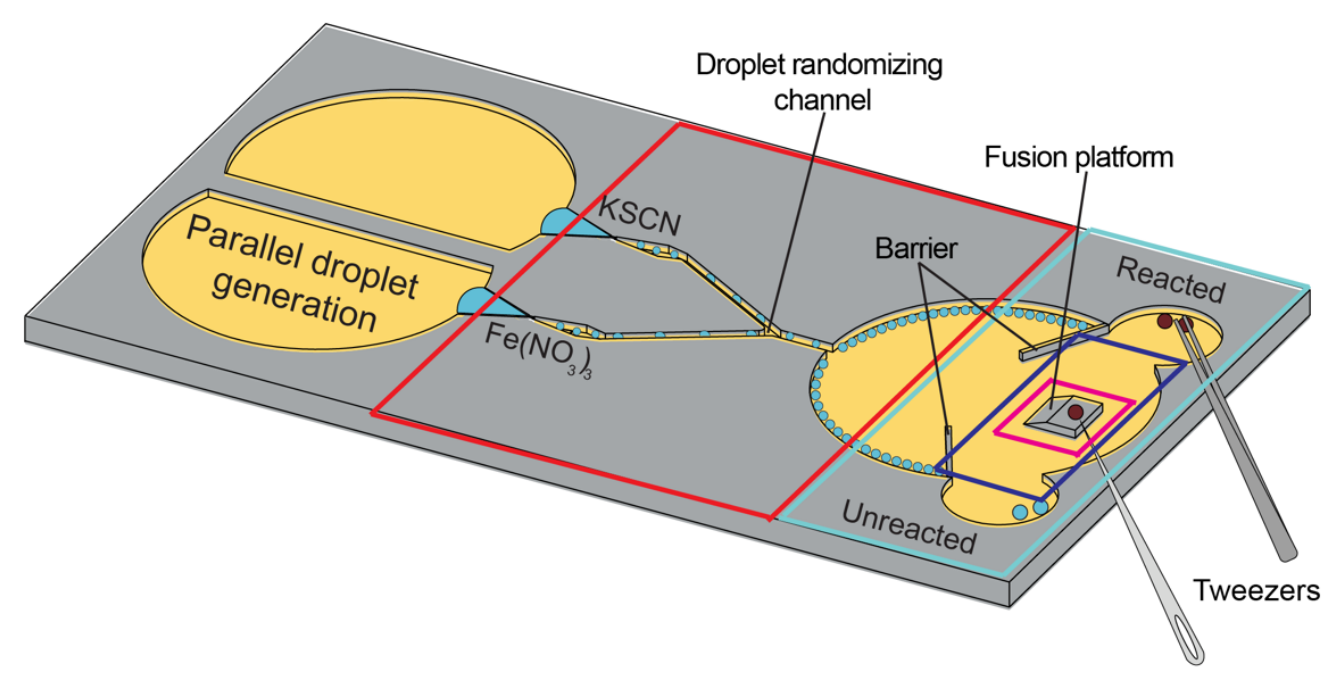

Needle

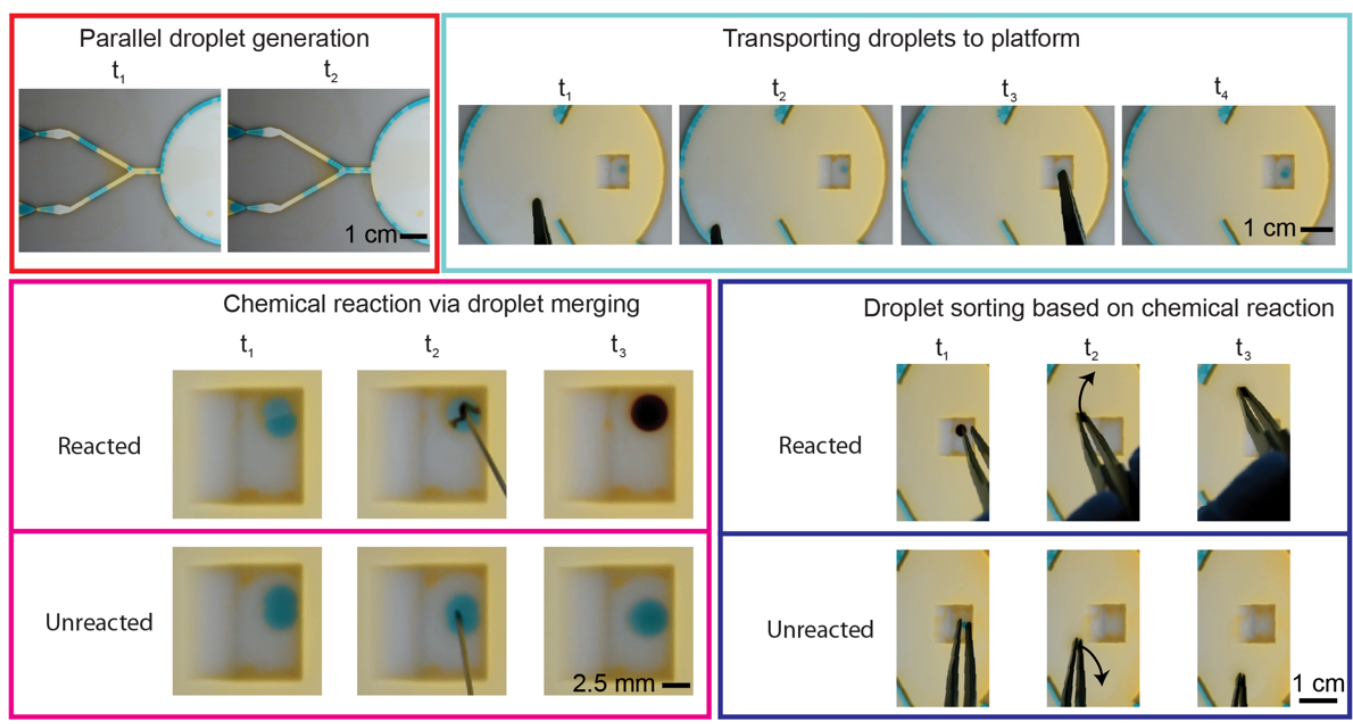

Figure 8. Model chemical reaction using capillary-driven open droplet microfluidics. Droplets containing $\mathrm{KSCN}$ and $\mathrm{Fe}\left(\mathrm{NO}_{3}\right)_{3}$ are generated in parallel. Two droplets are randomly selected and moved to the fusion platform with tweezers. The droplets are merged with a needle, if a colored complex $\left(\mathrm{Fe}(\mathrm{SCN})_{3}\right)$ forms, the droplet is sorted to the reacted chamber; droplets without $\mathrm{Fe}(\mathrm{SCN})_{3}$ are sorted to the unreacted chamber. Video available in Supplementary Video 7.

Overall, our open droplet microfluidic system is the first of its kind that can autonomously generate droplets by utilizing hydrostatic pressure and capillary pressure. Further, our open system enables manipulation of droplets (including patterning, transferring, splitting, merging, and sorting) due to unobstructed access to the droplets along the entire length of the device. This offers opportunities that simply are not possible in closed channel devices, where the ceiling obstructs access. We acknowledge that there are limitations of our system in comparison to traditional closed channel droplet-based microfluidics - chiefly, the speed of droplet formation and the number of droplets generated in a device is lower than in traditional systems where droplets are generated at thousands 
of droplets per second. Thus, we envision applications of our method will capitalize on aspects uniquely enabled by the open channel (such as the ability to manipulate droplets with implements like tweezers or a needle and the ability to add and remove solid objects like beads or pieces of tissue) that are suited for medium-throughput experiments (hundreds to thousands of droplets in total). Additionally, the ability to parallelize the channel without adding pumps to drive the flow allows for the droplet generation to be scaled up without adding additional equipment for each new droplet generator. Our method opens up numerous areas for future work-both on biological and chemical applications and in further understanding the physics underlying droplet formation during droplet generation and droplet transport via the Cheerios effect.

\section{Methods}

Channel design and fabrication. The channels were designed in Solidworks (Dassault Systemes SE) and postprocessed in Fusion 360 (Autodesk, Inc.). Then, they were fabricated out of $3.2 \mathrm{~mm}$ thick PTFE (McMaster-Carr Supply Co.) on a Datron NEO mill (Datron Dynamics Inc.). After the channels were milled, they were rinsed with deionized water (DIW), sonicated in $70 \%$ ethanol for $30 \mathrm{~min}$, and then rinsed again with DIW. Finally, the channels were dried with compressed air.

The channel height is $1.6 \mathrm{~mm}$, and the width of the narrow constriction ranges between $0.2 \mathrm{~mm}$ and $3.0 \mathrm{~mm}$. There is also a platform with a height of $0.2 \mathrm{~mm}$ at the narrow constriction and converging-diverging region to allow the aqueous plug to be pinned at the constriction entrance to produce consistent droplet generation. The channel has a square cross section. The converging part of the tapered channel is $45^{\circ}$, and diverging is $20^{\circ}$ as these angles were found to be the best at generating monodisperse droplets consistently. The square protrusions that form the channel constriction are $1 \mathrm{~mm}$ wide. CAD files and engineering drawings (SI Supplementary Note 4) are included in the SI.

General reagents. Carrier fluids used were pure HFE 7500 (The 3M Co.), HFE 7500 with 0.002 $\mathrm{wt} \%, 0.02 \mathrm{wt} \%, 0.2 \mathrm{wt} \%$, and $2 \mathrm{wt} \%$ Rhodamine fluorosurfactant (RAN Biotechnologies Inc.). The Rhodamine fluorosurfactant (FS) is a mix of fluorosurfactant 008FS (008-FluoroSurfactant) and Rhodamine-functionalized fluorosurfactant Rhod-FS (FS-Rhodamine) (FS is a mix of 008FS and Rhod-FS with weight ratio of 3:1 respectively). The aqueous phase, DIW, is mixed with blue food dye (McCormick Corp.).

Image acquisition setup. A Nikon-D5300 DSLR camera attached to a jig with adjustable distance was used to record the experimental results at the top view of the channel and at a frame rate of 30 fps. For the high-speed video in Figure 2b, a Chronos 1.4 high-speed camera (Kron Technologies Inc.) was installed on a stereoscope (United Scope LLC) to record the droplet generation at 2577 fps.

Droplet generation. A $180 \mu \mathrm{L}$ DIW plug containing food dye was pipetted on the platform in the channel and then $2 \mathrm{~mL}$ of carrier fluid was pipetted into the inlet reservoir of the channel to generate water droplets ranging from $0.52 \mu \mathrm{L}$ to $34.55 \mu \mathrm{L}$ (see Figure 1d). Generated droplet volume takes $1-2$ minutes to stabilize and generate consistent droplet volume.

Contact angle and interfacial tension measurements. Contact angles and interfacial tension values reported in Figure 3 were measured on a Kruss Drop Shape Analyzer model DSA025 (Kruss $\mathrm{GmbH})$. Contact angles of the aqueous droplet on the PTFE substrate were measured using the 
sessile droplet method. When needed, the sessile droplet method is conducted with the sessile droplet submerged in a second liquid to measure the contact angle in a quartz cuvette. In this experiment, the droplet was DIW with blue dye submerged in HFE7500 at various FS concentrations $(0 \mathrm{wt} \%, 0.002 \mathrm{wt} \%, 0.02 \mathrm{wt} \%, 0.2 \mathrm{wt} \%$, or $2 \mathrm{wt} \%)$ and the substrate was PTFE.

Interfacial tension was measured using the pendant drop method where the fluid body is deposited by a motorized syringe pump until it achieves a pendant shape. From deriving the force balance between the interfacial tension and gravity, the interfacial tension is extracted from the droplet shape. When needed, the pendant drop method is conducted with the pendant drop submerged in a second liquid to measure the interfacial tension in a quartz cuvette. In this experiment, the droplet was DIW with blue dye submerged in HFE7500 at various FS concentrations $(0 \mathrm{wt} \%, 0.002 \mathrm{wt} \%$, $0.02 \mathrm{wt} \%, 0.2 \mathrm{wt} \%$, or $2 \mathrm{wt} \%)$.

Droplet volume determination. A custom MATLAB script was written to determine the droplet volume. Using image processing, droplets were identified in the experimental videos and the area they encompass was calculated by counting the number of pixels of the droplet. From there, we calculated the effective diameter of the droplets from the area and depending on if droplet diameter was larger than channel height $\mathrm{h}$, the droplets were approximated as either spherical or cylindrical shape. When droplet diameter is less than the channel height, droplets have a spherical-like shape and when droplet diameter is larger than the channel height, droplets have a cylindrical-like shape. To calculate droplet volume:

If droplet diameter is larger than channel height $\mathrm{h}$,

$$
V=\pi r^{2} h
$$

If droplet diameter is smaller than channel height $h$,

$$
V=\frac{4}{3} \pi r^{3}
$$

$\mathrm{N} \geq 90$ droplets were analyzed per data point. The mean (red circle or blue square) and standard deviation (error bar) of the droplet volume are plotted in Figure 4.

Droplet sorting and merging (Figure 6 \& 7). For transporting a droplet, either a pair of tweezers or a PTFE bead attached to a stylus was used. The stylus was 3D-printed with grey resin (RS-F2GPGR-04, Formlabs Inc.) and has a 1/8" PTFE ball (McMaster-Carr Supply Co.) adhered to the tip with silicone sealant (Gorilla, Maine Wood Concepts Inc.). The tweezers were a pair of PTFE tweezers with slim, rounded, and smooth tips (McMaster-Carr Supply Co.). For merging droplets, a stainless steel stitching needle (AEHO crafts) or medical grade No. 22 hypodermic needle was used.

$\mathrm{KSCN}$ and $\mathrm{Fe}\left(\mathrm{NO}_{3}\right)_{3}$ aqueous solution preparation (Figure 8). Green food dye (McCormick Corp.) was added to solutions of $0.1 \mathrm{M}$ of KSCN (Thermo Fisher Scientific Corp.) and $0.05 \mathrm{M}$ of $\mathrm{Fe}\left(\mathrm{NO}_{3}\right)_{3}$ (Thermo Fisher Scientific Corp.) until they were approximately the same shade of blue. $\mathrm{KSCN}$ is clear while $\mathrm{Fe}\left(\mathrm{NO}_{3}\right)_{3}$ is yellow which is why the blue food dye was not added by exact measurements. The workflow of droplet generation, selection, fusion, and sorting (Figure 8) is described in the results section. 


\section{References}

1 Abate, A. R., Hung, T., Mary, P., Agresti, J. J. \& Weitz, D. A. High-throughput injection with microfluidics using picoinjectors. Proceedings of the National Academy of Sciences 107, 19163, doi:10.1073/pnas.1006888107 (2010).

2 Agresti, J. J. et al. Ultrahigh-throughput screening in drop-based microfluidics for directed evolution. Proceedings of the National Academy of Sciences 107, 4004, doi:10.1073/pnas.0910781107 (2010).

3 Doufène, K., Tourné-Péteilh, C., Etienne, P. \& Aubert-Pouëssel, A. Microfluidic Systems for Droplet Generation in Aqueous Continuous Phases: A Focus Review. Langmuir 35, 12597-12612, doi:10.1021/acs.langmuir.9b02179 (2019).

4 Seemann, R., Brinkmann, M., Pfohl, T. \& Herminghaus, S. Droplet based microfluidics. Rep Prog Phys 75, 016601, doi:10.1088/0034-4885/75/1/016601 (2012).

5 Shang, L., Cheng, Y. \& Zhao, Y. Emerging Droplet Microfluidics. Chemical Reviews 117, 7964-8040, doi:10.1021/acs.chemrev.6b00848 (2017).

6 Song, H., Chen, D. L. \& Ismagilov, R. F. Reactions in Droplets in Microfluidic Channels. Angewandte Chemie International Edition 45, 7336-7356, doi:https://doi.org/10.1002/anie.200601554 (2006).

7 Teh, S. Y., Lin, R., Hung, L. H. \& Lee, A. P. Droplet microfluidics. Lab Chip 8, 198-220, doi:10.1039/b715524g (2008).

8 Theberge, A. B. et al. Microdroplets in Microfluidics: An Evolving Platform for Discoveries in Chemistry and Biology. Angewandte Chemie International Edition 49, 5846-5868, doi:https://doi.org/10.1002/anie.200906653 (2010).

$9 \mathrm{Xi}, \mathrm{H} .-\mathrm{D}$. et al. Active droplet sorting in microfluidics: a review. Lab on a Chip 17, 751771, doi:10.1039/C6LC01435F (2017).

10 Yang, C.-G., Xu, Z.-R. \& Wang, J.-H. Manipulation of droplets in microfluidic systems. TrAC Trends in Analytical Chemistry 29, 141-157, doi:https://doi.org/10.1016/j.trac.2009.11.002 (2010).

11 Zhu, P. \& Wang, L. Passive and active droplet generation with microfluidics: a review. Lab on a Chip 17, 34-75, doi:10.1039/C6LC01018K (2017).

12 Berthier, E., Dostie, A. M., Lee, U. N., Berthier, J. \& Theberge, A. B. Open Microfluidic Capillary Systems. Analytical Chemistry 91, 8739-8750, doi:10.1021/acs.analchem.9b01429 (2019).

13 Berthier, J. \& Brakke, K. A. The Physics of Microdroplets. (Wiley, 2012).

14 Berthier, J., Brakke, K. A. \& Berthier, E. Open Microfluidics. (Wiley, 2016).

15 Casavant, B. P. et al. Suspended microfluidics. Proceedings of the National Academy of Sciences 110, 10111, doi:10.1073/pnas.1302566110 (2013).

16 Lee, J. J., Berthier, J., Kearney, K. E., Berthier, E. \& Theberge, A. B. Open-Channel Capillary Trees and Capillary Pumping. Langmuir 36, 12795-12803, doi:10.1021/acs.langmuir.0c01360 (2020).

17 Lee, J. J., Berthier, J., Theberge, A. B. \& Berthier, E. Capillary Flow in Open Microgrooves: Bifurcations and Networks. Langmuir 35, 10667-10675, doi:10.1021/acs.langmuir.9b01456 (2019).

18 Chen, L. et al. Elliptical Pipette Generated Large Microdroplets for POC Visual ddPCR Quantification of Low Viral Load. Analytical Chemistry 93, 6456-6462, doi:10.1021/acs.analchem.1c00192 (2021). 
19 Langer, K., Bremond, N., Boitard, L., Baudry, J. \& Bibette, J. Micropipette-powered droplet based microfluidics. Biomicrofluidics 12, 044106, doi:10.1063/1.5037795 (2018).

20 Lee, J. J. et al. Droplet Behavior in Open Biphasic Microfluidics. Langmuir 34, 5358-5366, doi:10.1021/acs.langmuir.8b00380 (2018).

21 Berry, S. B., Lee, J. J., Berthier, J., Berthier, E. \& Theberge, A. B. Droplet incubation and splitting in open microfluidic channels. Analytical Methods 11, 4528-4536, doi:10.1039/C9AY00758J (2019).

22 de Groot, T. E., Veserat, K. S., Berthier, E., Beebe, D. J. \& Theberge, A. B. Surface-tension driven open microfluidic platform for hanging droplet culture. Lab on a Chip 16, 334-344, doi:10.1039/C5LC01353D (2016).

23 Li, C., Boban, M. \& Tuteja, A. Open-channel, water-in-oil emulsification in paper-based microfluidic devices. Lab on a Chip 17, 1436-1441, doi:10.1039/C7LC00114B (2017).

24 Chowdhury, M. S. et al. Dendronized fluorosurfactant for highly stable water-influorinated oil emulsions with minimal inter-droplet transfer of small molecules. Nature Communications 10, 4546, doi:10.1038/s41467-019-12462-5 (2019).

25 Clausell-Tormos, J. et al. Droplet-based microfluidic platforms for the encapsulation and screening of Mammalian cells and multicellular organisms. Chem Biol 15, 427-437, doi:10.1016/j.chembiol.2008.04.004 (2008).

26 Soitu, C. et al. Raising fluid walls around living cells. Science Advances 5, eaav8002, doi:10.1126/sciadv.aav8002 (2019).

27 Soitu, C. et al. Microfluidic chambers using fluid walls for cell biology. Proceedings of the National Academy of Sciences 115, E5926, doi:10.1073/pnas.1805449115 (2018).

28 Soitu, C. et al. Jet-Printing Microfluidic Devices on Demand. Advanced Science 7, 2001854, doi:https://doi.org/10.1002/advs.202001854 (2020).

29 Walsh, E. J. et al. Microfluidics with fluid walls. Nature Communications 8, 816, doi:10.1038/s41467-017-00846-4 (2017).

$30 \mathrm{Li}, \mathrm{C}$. et al. Under oil open-channel microfluidics empowered by exclusive liquid repellency. Science Advances 6, eaay9919, doi:10.1126/sciadv.aay9919 (2020).

31 Li, C., Niles, D. J., Juang, D. S., Lang, J. M. \& Beebe, D. J. Automated System for SmallPopulation Single-Particle Processing Enabled by Exclusive Liquid Repellency. SLAS Technol 24, 535-542, doi:10.1177/2472630319853219 (2019).

$32 \mathrm{Li}, \mathrm{C}$. et al. Double-exclusive liquid repellency (double-ELR): an enabling technology for rare phenotype analysis. Lab on a Chip 18, 2710-2719, doi:10.1039/C8LC00584B (2018).

33 Li, C. et al. Exclusive Liquid Repellency: An Open Multi-Liquid-Phase Technology for Rare Cell Culture and Single-Cell Processing. ACS Applied Materials \& Interfaces 10, 17065-17070, doi:10.1021/acsami.8b03627 (2018).

34 de Rutte, J., Dimatteo, R., van Zee, M., Damoiseaux, R. \& Di Carlo, D. Massively parallel encapsulation of single cells with structured microparticles and secretion-based flow sorting. bioRxiv, 2020.2003.2009.984245, doi:10.1101/2020.03.09.984245 (2020).

35 Baroud, C. N., Gallaire, F. \& Dangla, R. Dynamics of microfluidic droplets. Lab on a Chip 10, 2032-2045, doi:10.1039/C001191F (2010).

36 Gai, Y., Khor, J. W. \& Tang, S. K. Y. Confinement and viscosity ratio effect on droplet break-up in a concentrated emulsion flowing through a narrow constriction. Lab on a Chip 16, 3058-3064, doi:10.1039/C6LC00478D (2016).

37 Vella, D. \& Mahadevan, L. The "Cheerios effect". American Journal of Physics 73, 817825, doi:10.1119/1.1898523 (2005). 
38 Lucassen, J. Capillary forces between solid particles in fluid interfaces. Colloids and Surfaces 65, 131-137, doi:https://doi.org/10.1016/0166-6622(92)80268-7 (1992).

39 Chan, D. Y. C., Henry, J. D. \& White, L. R. The interaction of colloidal particles collected at fluid interfaces. Journal of Colloid and Interface Science 79, 410-418, doi:https://doi.org/10.1016/0021-9797(81)90092-8 (1981).

40 Ho, I., Pucci, G. \& Harris, D. M. Direct Measurement of Capillary Attraction between Floating Disks. Physical Review Letters 123, 254502, doi:10.1103/PhysRevLett.123.254502 (2019).

41 Stamou, D., Duschl, C. \& Johannsmann, D. Long-range attraction between colloidal spheres at the air-water interface: the consequence of an irregular meniscus. Phys Rev E Stat Phys Plasmas Fluids Relat Interdiscip Topics 62, 5263-5272, doi:10.1103/physreve.62.5263 (2000).

\section{Acknowledgments}

We gratefully acknowledge funding from the National Institutes of Health (NIH) through National Institute of General Medical Sciences award number R35GM128648, the Arnold and Mabel Beckman Foundation (Beckman Young Investigator Award), the David and Lucile Packard Foundation (Packard Fellowship for Science and Engineering), and the Society for Laboratory Automation and Screening (SLASFG2020, UNL). Any opinions, findings, and conclusions or recommendations expressed in this material are those of the author(s) and do not necessarily reflect those of the Society for Laboratory Automation and Screening or the NIH.

\section{Author contributions}

J.K., U.N.L., J.B., E.B., and A.B.T. designed the research; J.K. and J.B. discussed and derived theory; J.K. and U.N.L. performed experiments; and J.K., U.N.L., J.B., E.B., and A.B.T. interpreted the results and wrote the paper.

\section{Competing interests}

A.B.T. has ownership in Stacks to the Future, LLC and E.B. has ownership in Stacks to the Future, LLC, Tasso, Inc., and Salus Discovery, LLC. However, this research is not related to these companies. 